\title{
La teoría de redes en las investigaciones jurídicas: una revisión de la literatura
}

\author{
The Network Theory in Legal Investigations: A Review \\ of the Literature \\ A teoria das redes em investigações jurídicas: uma revisão \\ da literatura
}

\section{Diana Alexandra Castañeda Guerrero*}

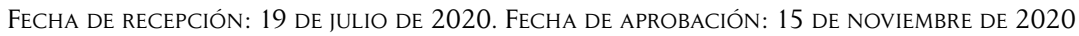

Doi: https://doi.org/10.12804/revistas.urosario.edu.co/sociojuridicos/a.9359

Para citar este artículo: Castañeda Guerrero, D. A. (2021). La teoría de redes en las investigaciones jurídicas: una revisión de la literatura. Revista Estudios Socio-Jurídicos, 23(2), 273-301. https://doi.org/10.12804/revistas.urosario. edu.co/sociojuridicos/a.9359

\section{RESUMEN}

La investigación jurídica contemporánea se enfrenta al reto de incorporar metodologías utilizadas en otras ciencias sociales, es en ese escenario que aparece la teoría de redes como un instrumento que aporta herramientas para el abordaje del estudio del derecho. Existen tres aplicaciones del análisis de redes: las redes de normas, las redes jurisprudenciales y las redes textuales para analizar la estructura interna de una norma. Luego de presentar las tres perspectivas como una forma de acercar a otros investigadores al uso de las redes en las ciencias jurídicas, se manifiesta que la potencialidad de las redes en el manejo de grandes cantidades de información puede convertirse en una limitación por la incapacidad de observar los detalles de los objetos de estudio.

Palabras clave: teoría de redes; redes legales; redes jurisprudenciales.

* Universidad Nacional de Colombia. Abogada, Especialista en Derecho Administrativo, Magister en Ciencias Económicas y Doctora en Derecho. Correo electrónico: dacastanedag@ unal.edu.co. ORCID: https://orcid.org/0000-0003-3010-8242 


\section{ABSTRACT}

Contemporary legal research faces the challenge of incorporating methodologies used in other social sciences; it is in this scenario that Network Theory appears as an instrument that provides tools in the approach to the study of law. There are three applications for network analysis, norm networks, jurisprudential networks, and textual networks to analyze the internal structure of a norm. After presenting the three perspectives as a way to bring other researchers closer to the use of networks in legal sciences, they state that the potential of networks in handling large amounts of information can become a limitation due to the inability to observe the details of the objects of study.

Keywords: Network theory; legal networks; jurisprudential networks.

\section{RESUMO}

A pesquisa jurídica contemporânea enfrenta o desafio de incorporar metodologias utilizadas em outras ciências sociais, e é neste cenário que a Teoria das Redes surge como um instrumento que fornece ferramentas na abordagem para o estudo do direito. Existem três aplicações que foram dadas à análise de redes, redes de normas, redes jurisprudenciais e redes textuais para analisar a estrutura interna de uma norma. Após apresentar as três perspectivas como forma de aproximar outros pesquisadores ao uso de redes nas ciências jurídicas, afirma-se que o potencial das redes em lidar com grandes quantidades de informação pode se tornar uma limitação pela impossibilidade de observar os detalhes dos objetos de estudo.

Palavras-chave: teoria das redes; redes jurídicas; redes jurisprudenciais. 
El uso de la teoría de redes en los estudios del derecho es un campo que ofrece múltiples posibilidades y tiene una potencialidad pendiente de ser explorada. Sin embargo, entre los juristas colombianos no se ha dimensionado en su justa medida. En este artículo se demuestra que hay aplicaciones fructíferas del análisis de redes en la academia jurídica. Como se demostrará, a lo largo del siglo XXI ha existido una importante aplicación del análisis de redes en las investigaciones jurídicas. Aquí se busca contestar la pregunta: ¿cuál ha sido el aporte de la teoría de redes a la investigación jurídica?

Este artículo se encuentra dividido en tres secciones. En la primera parte se realizará una sucinta presentación de la teoría de redes (TdR), seguido de los desarrollos que se han realizado en estudios de tipo jurídico, donde se presentarán tres perspectivas: la del sistema jurídico visualizado como una de red de normas, las redes jurisprudenciales y las redes para observar la estructura interna de la legislación. Finalmente, se presentarán los límites y potencialidades de la investigación jurídica utilizando la TdR.

\section{Introducción a la teoría de redes}

La TdR se construyó como un método de estudio de distintos tipos de sistemas o grupos de elementos. El interés del enfoque de redes es examinar la estructura global de interacciones de un sistema e ignorar las propiedades particulares de cada elemento (Caldarelli \& Catanzaro, 2012). Las aplicaciones de la TdR van desde redes ecológicas, sociales, financieras, tecnológicas, informáticas, económicas y sociológicas hasta cualquier tipo de colectividad que posea una multiplicidad de elementos, ${ }_{1}^{1}$ donde sea importante observar el comportamiento en conjunto.

La colectividad bajo estudio se representa en un grafo $^{2}$ que es la presentación gráfica de un conjunto de nodos (vértices) unidos por

1 Respecto a la clasificación se puede ver Newman (2010), quien clasifica las redes en tecnológicas, sociales, de información y biológicas.

2 En el origen de la Teoría de Redes se encuentra la teoría de grafos, que define un grafo como "un conjunto, no vacío de objetos llamados vértices (o nodos) y una selección de pares de vértices, llamados aristas (edges) que pueden ser orientados o no. (...) El estudio de 
conexiones (aristas o $\operatorname{arcos})^{3}$ que evidencian las relaciones, de manera que se logra una visión global de la red analizada. El estudio de un sistema desde la TdR permite observar sistemas complejos desde una perspectiva amplia, especialmente cuando hay numerosos datos para analizar, debido a que permite observar "la arquitectura oculta de la complejidad" (Caldarelli \& Catanzaro, 2012, p. 18).

El enfoque de la TdR busca explicar la manera en que un conjunto de elementos se convierte en una comunidad, identificando el patrón de interacciones y aportando nueva información para el análisis del comportamiento colectivo de los elementos estudiados. Así, la TdR permite conocer cuáles son las propiedades escondidas bajo la estructura que puede no ser visible. Aunque esta es un campo relativamente nuevo que heredó métodos de la física (redes complejas) y de la matemática (teoría de grafos) para enfrentar la cantidad de información que generaban redes sociales como Twitter o Facebook, los métodos utilizados empiezan a generar aplicaciones para analizar problemas de investigación en materia jurídica de diferentes maneras. A finales del siglo XX empezaron a hacerse visibles las investigaciones en esta área como el trabajo pionero de Bench-Capon et al. (1997).

Al ser la TdR un campo novedoso en la investigación jurídica es necesario realizar una introducción al lector de los conceptos básicos que se van a utilizar para hacer comprensible la literatura revisada en este artículo.

En primer lugar, es necesario delimitar el concepto de nodos, entendidos como los distintos tipos de elementos que se relacionan y son el objeto de estudio en una investigación. La especificación de lo que se tome como nodo dependerá de las características de la investigación planteada, por ello "pueden representar entidades individuales (personas, ordenadores, especies, genes...) que intercambian material o información (paquetes de información, energía, etc.), o pueden representar

\footnotetext{
las redes se ocupa de los gráficos, entendidos como una representación de cualquiera de las relaciones simétricas o (más generalmente) asimétricas, entre los objetos discretos"(Arjona Sánchez, 2010, p. 116).

3 Las aristas evidencias las relaciones entre nodos de manera no orientada (grafos no orientados), mientras que los arcos muestran pares ordenados de nodos, en donde se evidencia la dirección del enlace entre uno y otro vértice.
} 
ubicaciones (países, aeropuertos...) que intercambian entidades individuales (mercancías, pasajeros...)" (Caldarelli \& Catanzaro 2012, p. 137). Por esto, la TdR es plenamente compatible con la investigación jurídica y su aplicabilidad va a depender de las definiciones; las posibilidades que ofrece al estudio del derecho son múltiples.

Un ejemplo de una red simple se puede observar en la figura 1: tres nodos interconectados por igual número de bordes.

Figura 1. Ejemplo de red

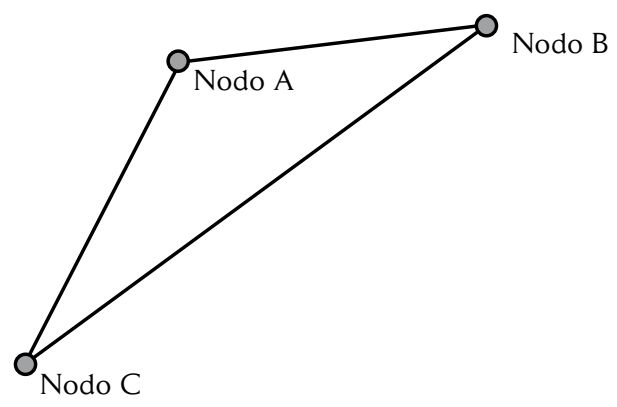

\section{Estudios jurídicos utilizando la TdR}

La relación entre la TdR y la investigación jurídica ha sido analizada y aplicada desde varios puntos de vista, en algunos casos corresponde directamente con el interior del sistema jurídico, allí los nodos son normas relacionadas entre sí (como lo hacen Boella et al., 2014) o sentencias judiciales (por ejemplo, el estudio de Fowler et al., 2007). Tambien se ha utilizado la TdR para observar problemas asociados a situaciones del entorno de la enseñanza o práctica del derecho como la relación entre facultades de derecho estadounidenses (acá se destaca el estudio de Martin Katz \& Bommarito, 2011), de organizaciones (Sweeney et al., 2014) o las redes criminales en la investigación penal (Lettieri et al. 2014a; Lettieri et al., 2014c). Esto demuestra que, a pesar de que los objetivos de cada investigación son variados, en general se busca simplificar la complejidad de la gran cantidad de datos que involucra la investigación de los sistemas jurídicos. 
Para Gultemen y Van Engers (2014) la literatura de teoría de los grafos que aparece involucrada en los estudios del derecho puede ser dividida en dos: macronivel y micronivel. El macronivel se encarga de los análisis de la estructura general de una red de citación legal, incluyendo casos, estatutos y otras autoridades legales como lo realizaron Fowler et al. (2007), Smith (2005), Mazzega et al. (2009), Boulet et al. (2011) y Winkels et al. (2011). Por su parte, el micronivel está concentrando en la granularidad, los aspectos lógico-semánticos y la precisión en la generación de nodos conectados, en este caso los nodos están dados por unidades significativas de los textos (objetos textuales) vinculados con los bordes de las relaciones lógicas entre ellos; los autores lo denominan estudio de la estructura granular de los textos legales, que son representados en gráficos o figuras, como lo hacen Winkels y Boer (2014).

Para demostrar la compatibilidad entre los problemas de investigación de connotación jurídica y la $\mathrm{TdR}$, se presentarán a continuación tres grandes grupos de investigaciones realizadas en esta materia: la visualización de la estructura interna de los sistemas jurídicos, las redes jurisprudenciales y las redes al interior de textos normativos.

\section{El sistema jurídico como una red}

Hay variados estudios que encuentran similitud entre los sistemas jurídicos y una gran red de información, en ellos la TdR sirve para repensar o revisar la estructura constitucional y legal de los Estados modernos, en donde las normas se relacionan de diferentes formas y se producen constantemente, desdibujando la tradicional pirámide normativa.

En este contexto, Augsberg (2009) realiza un análisis de la importancia de los modelos de redes en el discurso jurídico, partiendo de la pregunta de si vale la pena involucrar el concepto de redes en el derecho; al respecto considera que la TdR permite evidenciar un acto de integración entre distintos elementos como en el caso de los procedimientos transnacionales de unificación de leyes de la Unión Europea. Además, la TdR constituiría una herramienta que puede contribuir a dar respuesta a problemas específicos de índole sociológico y filosófico. Sociologicamente, la red constituye la respuesta para estudiar la sociedad moderna en el marco de la globalización, en donde el papel 
de los sistemas legales es relevante. Fisolóficamente, el pensamiento en red propone una visión de poliperspectivismo, en donde se estudia la coordinación de relaciones anónimas.

Aparece la pregunta en Augsberg (2009) de si existe una necesidad epistemológica de buscar una forma de pensamiento distinta a la deductiva-racional que tenga un enfoque transversal-interrelacionado, como lo posibilita la TdR. Este escenario es muy útil en situaciones donde coexisten normas nacionales y supranacionales. El hecho de que el concepto red sea exógeno al contexto jurídico genera prevención por parte de los juristas acostumbrados al concepto de jerarquía piramidal kelseniana; sin embargo, puede contribuir al estudio contemporáneo de problemas jurídicos.

En ese mismo sentido, Arjona Sánchez (2010) realiza una explicación del derecho en red como una nueva propuesta para analizar la complejidad del derecho contemporáneo, al respecto afirma que el positivismo jurídico se encuentra en crisis a raíz de los cambios en el mundo jurídico. Dentro de los cambios, anota el surgimiento del ciberespacio como un nuevo objeto de estudio del derecho, debido a las numerosas relaciones jurídicas que surgen en este escenario entre individuos alrededor del mundo, lo que desplaza la soberanía de los Estados por los operadores que controlan las redes. A raíz de esto se propone una nueva categoría: el derecho en red como una transformación del derecho piramidal.

La crisis del derecho piramidal kelseniano se observa si se tiene en cuenta que la visión escalonada de los ordenamientos jurídicos resulta insuficiente para mostrar la complejidad del derecho contemporáneo, debido a la multiplicidad de fuentes nacionales e internacionales que generan pluralidad de sistemas normativos que coexisten en un territorio (Arjona Sánchez, 2010). En ese escenario, la TdR en el estudio del derecho es plenamente pertinente, advirtiendo que en el campo jurídico no ha sido suficientemente explorada y aunque "para muchos juristas lo que no está claro, es si el modelo [de redes] tiene un valor meramente estético o por el contrario forma parte de un descubrimiento científico relevante" (Arjona Sánchez, 2010, p. 98), se considera que la red es más que una gráfica que permite mover las fronteras del conocimiento jurídico. La metáfora de pensar el derecho en forma jerárquica corresponde a la habilidad del conocimiento humano de representar 
visualmente los espacios y tiempos, frente a lo que se presenta una evolución modelando los espacios en forma de red que corresponde más a un espacio de flujos. Los flujos corresponderían a la estructura de la sociedad, donde alternan de manera permanente el capital, la información, el conocimiento, etc., y en donde vale la pena estudiar los elementos que permiten la circulación de información legal.

Arjona Sánchez (2010) identifica una preocupación común en varios textos que combinan derecho y redes, que gira en torno al problema de la centralidad, ${ }^{4}$ que en la TdR es la información respecto a la importancia relativa de los nodos de un gráfico, de forma tal que puede medirse la centralidad de un elemento en una red que en principio no tenía un centro previamente definido. Así, en aquellos casos en donde la relación entre normas o conjuntos de normas no puede ser fácilmente explicada en la lógica de la piramide kelseniana, preguntarse por la centralidad permitiría entender la lógica del ordenamiento jurídico contemporáneo, debido a que unos nodos serán más visitados que otros en la topología de una determinada red jurídica; es decir, no se ubica la cúspide de una piramide sino unos nodos en donde la aglutinación muestra mayor relevancia. Por ejemplo, en la figura 2, se puede observar la centralidad del nodo A, que tiene siete conexiones con los otros nodos.

Figura 2. Ejemplo de red visualizando la centralidad del nodo A

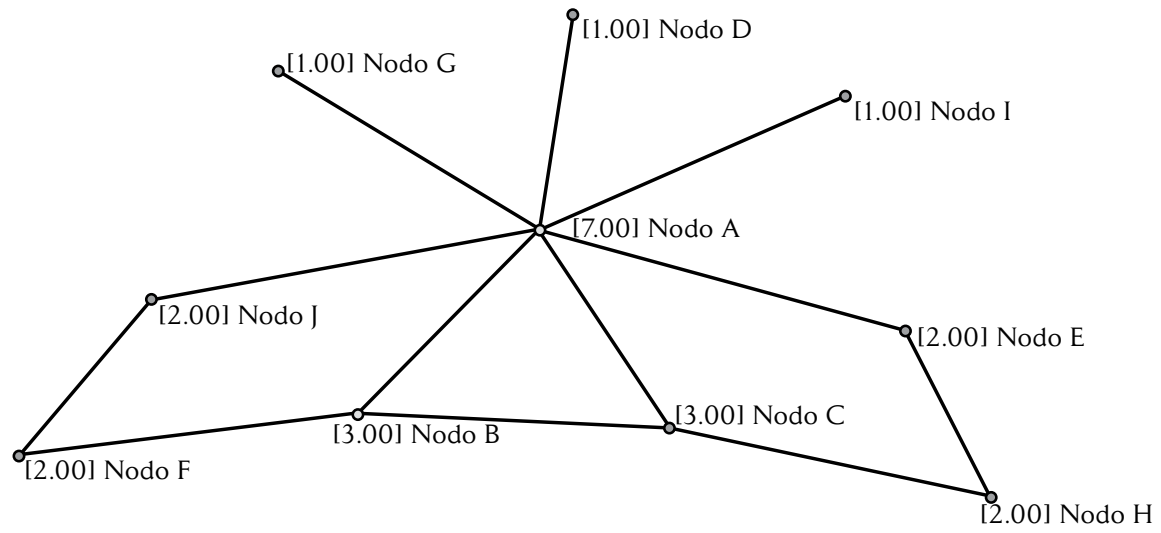

4 El grado de centralidad de un nodo es definido como el número de bordes o arcos adyacentes a este nodo, es una manera de medir su importancia. 
De esta forma queda evidenciado que la TdR ofrece distintas perspectivas al estudio del derecho, en particular, en cuestiones donde la descripción piramidal se ha quedado corta, como un punto de partida en la búsqueda de soluciones de nuevos problemas que se plantean en la investigación jurídica:

creemos honestamente que la teoría de redes, (...) puede iluminarnos en un camino que debe hacer por si misma la ciencia del Derecho, apoyada, eso sí, en los conocimientos de otras ramas del saber científico, pero al fin y al cabo de forma autónoma (Arjona Sánchez, 2010, p. 141).

Es decir, se cree que la explicación del ordenamiento jurídico en una estructura de piramide explica solamente una parte del derecho contemporáneo, pero no lo refleja en su totalidad:

[1] que se queda fuera de la pirámide es, con frecuencia, precisamente lo más nuevo. Por eso la transformación profunda del derecho actual obliga a un cambio de paradigma en su estudio. El modelo de la red sustituye al de la pirámide (Losano, 2005, p. 180).

El autor cree que la teoría pura del derecho (Kelsen, 1982) está atada a la noción de Estado nacional moderno, por lo que no explica satisfactoriamente lo que sucedía antes, en el derecho preindustrial donde no había un Estado con las posibilidades de instaurar las normas jurídicas para la totalidad de la sociedad ni explica el derecho postindustrial que se presenta con la existencia del pluralismo jurídico. Sin embargo, Losano (2005) mostrará algo de desconfianza hacia la representación en red de los ordenamientos jurídicos.

Para defender la aplicabilidad de la TdR los mejores argumentos son las aplicaciones empíricas, que el texto de Arjona Sánchez (2010) no defiende, simplemente desarrolla la defensa teórica de las posibilidades de aplicación al derecho constitucional de la Unión Europea como un ejemplo de derecho en red, porque ejemplifica la falta de jerarquía normativa y la existencia de lo que denomina flujos jurídicos. La visión del derecho en red es diferente a la del derecho escalonado kelseniano, pero no la sustituye por completo, la evoluciona. 
Los estudios de redes legales se han aplicado a varios sistemas jurídicos por académicos de distintos países del mundo: en Francia por Mazzega et al. (2009), Boulet et al. (2011) y Mimouni et al. (2014); en Italia por Boella et al. (2014); en Estonia por Täsk et al. (2011); en Holanda por Hoekstra (2014); en Hungría por Szöke et al. (2014); en Turquía por Gultemen y Van Engers (2014), y en Alemania, Winkels y Boer (2014).

Todos estos autores coinciden con lo expresado por Ashley et al. (2014), quienes consideran que el análisis legal tradicional es una herramienta para interpretar estatutos y regulaciones, pero no para medir las relaciones dentro de los sistemas jurídicos. Para ello es muy útil acudir al análisis de redes, en donde se pueden describir las características de la red, analizar patrones complejos de relación y producir resultados gráficos, de forma que permite complementar el análisis legal tradicional que se encarga de discernir el contenido sustancial de las normas legales.

Por consiguiente, luego de identificar el tipo de relación que se quiere representar gráficamente y de obtener la base de datos y la imagen correspondiente a la estructura del sistema jurídico, Garbarino (2014) considera que la facultad de visualizar el sistema jurídico como una red hace posible identificar una estructura en distintos momentos del tiempo, como una especie de fotografía del sistema jurídico, que posibilita estudiar la evolución de su estructura.

Los autores que realizan estudios jurídicos con aplicación de la TdR identifican las conexiones entre las normas como lo hace el texto de Tamme et al. (2014), citado por Zhang et al. (2014), en donde se muestra dependencia entre normas del derecho estonio, a partir de las cuales se pueden realizar clasificaciones de importancia de las normas y simular, por ejemplo, el impacto normativo que tendría la derogatoria de alguna de las normas interrelacionadas.

Generalmente estas redes de normas se realizan a partir de "cadenas de producción", en donde una regla genera la siguiente y de esta forma resultan enlazados (por medio de arcos) los nodos (reglas en este caso), generando los eslabones de la red, que puede ser dibujada y muestra la producción de reglas a través del tiempo. Dicho de otra forma, los diagramas representan la evolución de una red de reglas en una dimensión temporal que permite hacer seguimiento a la secuencia de producción. 
La utilidad del trazado de redes normativas es doble, en primer lugar, demuestra que los sistemas jurídicos tienen una estructura identificable en cada periodo y, en segundo, permite trazar su estructura evolutiva. La evolución, entendida como el conglomerado de cambios dentro del sistema, es una característica de la estructura en red que representa la producción de reglas representadas por enlaces de producción y describe cómo se desarrolla el cambio legal dentro de una estructura de $\operatorname{red}^{5}$ (Garbarino, 2014), debido a que las redes son intrínsecamente dinámicas y no pueden ser estudiadas como estructuras estáticas; de la misma manera en que se conectan las redes de telecomunicaciones, las redes jurídicas se modifican cuando aparecen nuevos nodos. ${ }^{6}$

En el mismo sentido, Garbarino (2014) considera que el coeficiente de agrupamiento ${ }^{7}$ sirve para estudiar las redes jurídicas ya sea de normas o de sentencias judiciales en países del sistema de common law porque en este caso muestra el nivel de conexidad entre los casos que son resueltos bajo la regla del stare decisis. Sin embargo, aclara que no necesariamente todas las citaciones generan dependencia, por lo que es necesario que se verifique la existencia de un vínculo real entre el caso que influye y el influenciado.

Desde esta visión, los sistemas jurídicos son vistos como una red compleja en donde se entrelazan reglas producidas por una amplia gama de agentes y tienden a funcionar de forma agrupada en donde los nodos no están todos uniformemente ligados entre sí, sino que algunos están densamente conectados en grupos, denominados clústeres, detectados mediante el coeficiente de agrupamiento. A partir de ello se puede encontrar, según Garbarino (2014), que hay cadenas en la red que producen mayores variaciones normativas en distintos momentos históricos; a su vez, los nodos con mayor centralidad, que operan como importantes conectores, tienden a ser de un número reducido, lo que

5 Estos cambios al interior de las redes normativas ocurren porque se añaden o eliminan nuevos nodos (reglas).

6 En algunos casos se observa que hay nodos que tienen una tendencia a atraer más conexiones, lo que se conoce como un "apego preferencial", mientras que hay otros que permanecen desconectados.

7 El coeficiente de agrupamiento o coeficiente de transitividad de un vértice en un grafo calcula que tan interconectado se encuentra con los otros vértices. 
permite sacar conclusiones relacionadas con la evolución del sistema jurídico analizado y del comportamiento de los agentes creadores de las normas.

Por otra parte, es importante destacar que el estudio de las redes es intrínsecamente una herramienta empírica, solo la teoría no prueba su utilidad, es por ello que el alcance de un estudio de redes está definido por el conjunto de preguntas de investigación que se realicen para su diseño -especialmente, cuáles son los nodos y qué tipo de enlaces tienen-, lo que guía la recopilación de datos y el desarrollo para modelar el sistema. De hecho, la TdR inició con el estudio de sistemas fácilmente observables entre individuos en pequeños grupos sociales y luego se extendió a sistemas mucho más complejos con multiplicidad de nodos, para los cuales se hizo necesario acudir al uso de herramientas informáticas para identificarlos, relacionarlos y poder crear representaciones de la red basadas en principios estadísticos. En relación con esto, Garbarino (2014) reitera que los sistemas jurídicos vistos como redes de reglas (nodos) que evolucionan continuamente son sin duda objetos complejos; no obstante, la tarea de identificar realmente las reglas para modelar estas redes y sus cambios se puede lograr porque los datos legales actualmente se almacenan en bases de datos electrónicas, en consecuencia la recopilación material de datos legales, aunque pueda parecer desalentadora, es similar a la de otras áreas (por ejemplo, redes de citas) y, por tanto, es factible si las guías de discernimiento que guían esta recolección se extraen correctamente. Además, se debe tener en cuenta qué tipo de enlaces entre las reglas debe ser incluído para guiar la recolección de tales datos, allí se considera que los vínculos de producción son las relaciones esenciales entre las normas que deben contener en un modelo del sistema jurídico; para demostrar su disertación Garbarino (2014) procedió a trazar la red del sistema tributario estadounidense.

En ese sentido, es pertinente presentar algunas de las investigaciones que han realizado aplicaciones de la TdR a distintos sistemas jurídicos. Dentro de ellas aparece el texto de Mazzega et al. (2009) que, siguiendo lo anunciado en Bourcier \& Mazzega (2007), contiene un análisis de leyes codificadas en Francia como un sistema estructurado, para ello incluyeron 52 códigos que se toman como nodos y la citación mutua 
como las conexiones de una gran red legal. Analizadas las propiedades de la red, a partir de la medida de centralidad, encontraron que hay un grupo de diez códigos que son los más citados, además de otros tres grupos de códigos que son dominantes en sus áreas particulares; concluyen que este tipo de perspectiva abre nuevas posibilidades en la caracterización científica y medición de la dimensión de la complejidad jurídica.

De otro lado, un estudio similar se encuentra en Boella et al. (2014) que presenta un análisis ampliado de una gran red de leyes italianas, proporcionando varios tipos de análisis que pueden ser utilizados dentro de un sistema de gestión legal para la comprensión de los datos. Mencionan que para el uso de las herramientas tecnológicas que son útiles en la aplicación de la TdR en el derecho se debe tener una buena comprensión de la estructura regulatoria; es decir, los software ayudan pero son los conocimientos y la habilidad del investigador los que van a permitir que las redes trazadas tengan sentido teórico, aunque siempre va a aparecer un elemento de subjetividad en la interpretación que tienen las normas jurídicas, en el trazado de las relaciones que se están dibujando; sin embargo, la complejidad de las cadenas normativas se puede simplificar con el uso de las herramientas tecnológicas adecuadas.

Desde una perspectiva similar, se encuentra el texto de Täsk et al. (2011) quienes realizan una visualización en redes de la legislación de Estonia, para representar la complejidad existente en su sistema jurídico. En esta investigación se evidencia la utilidad de la representación gráfica de la legislación. Los autores propusieron una descomposición estructural del texto en palabras y cláusulas lingüísticas, como componentes básicos de todas las normas jurídicas, con ellos probaron la teoría propuesta al comparar la conformación interna de distintas leyes y encontraron que existen estructuras ocultas a la simple lectura e interpretación tradicionalmente realizada por los abogados cotidianamente.

Bajo el enfoque de representar de forma simplificada la complejidad de un sistema jurídico, Boulet et al. (2011) realizaron un estudio más amplio que el anterior, en el cual involucraron todos los códigos franceses, con el fin de examinar la interdependencia entre ellos, para tal efecto tomaron cada código como un vértice y la citación entre ellos, al menos una vez, como una arista. A partir de la representación gráfica 
de la red, los autores consiguieron determinar cuáles son los códigos más influyentes en el sistema jurídico francés al momento de realizar el trazado del grafo. En este caso, se encontró que el comportamiento de la red es de tipo pequeño mundo; ${ }^{8}$ aunque tiene solo 51 vértices, el número de códigos del sistema jurídico francés, no tiene una baja densidad, debido a que tiene 531 conexiones o bordes, lo que es tomado por los autores como un número importante que le da robustez al grafo.

Por su parte, Hoekstra (2014) aplicó la TdR para representar toda la regulación holandesa con el propósito de hacer redes de datos legales. El autor retomó el trabajo realizado por Bommarito y Katz (2010), enfatizando que el trazado de la red permite determinar cuáles son las normas más influyentes e importantes del sistema jurídico analizado mediante la medida de centralidad, que determina el nodo con mayor cantidad de conexiones. Simultáneamente, Szöke et al. (2014) realizaron un ejercicio similar al de Hoekstra (2014), pero aplicado al derecho húngaro, y proponen un marco de extracción automática de conocimientos que convierte un documento legal tomado como un corpus en una base de conocimiento multidominio que permite analizar los metadatos y relaciones inherentes en el gran corpus del sistema jurídico. De la misma forma, Gultemen y Van Engers (2014) utilizan la TdR para procesar la legislación turca y estudiar la consistencia del sistema jurídico desde sus fuentes y las interacciones interregulatorias que ocurren entre ellas. En estos trabajos se enfatiza la importancia y la dificultad de la recuperación de los datos legales para trazar las redes jurídicas.

De otro lado, Mimouni et al. (2014) estudian la referencia entre fuentes del derecho en la legislación francesa, partiendo del punto que en la actualidad ningún documento debe ser interpretado de manera aislada; consideran que la visión en red del sistema jurídico es muy acertada por lo enmarañadas que pueden parecer las fuentes del derecho y los constantes cambios que ocurren en cada fuente, por ello realizan un ejercicio empírico para demostrar la pertinencia de su propuesta en el cual relacionan leyes francesas con actos administrativos locales.

8 Se llama una red de pequeño mundo a la representada en un grafo donde la mayoría de los nodos no están conectados directamente, pero pueden contactarse por medio de un trayecto corto atravesando otros nodos. Además, la probabilidad de que dos vértices estén unidos por un borde es mayor si estos ya tienen un vecino en común (Watts E Strogatz, 1998). 
Finalmente, entre los textos empíricos que realizan aplicaciones interesantes de la TdR en el derecho se destaca el texto de Boella et al. (2014) que busca solucionar de manera aplicada el problema de la gran red legislativa que interactúa en la Unión Europea, similar al ejercicio teórico realizado por Arjona Sánchez (2010), quien se basa en la taxonomía legal realizada por Ajani et al. (2008), en este caso utilizando las relaciones por las referencias entre leyes para trazar las redes. Este método lo encontraron útil debido a la multiplicidad de fuentes que tiene el derecho italiano, allí identificaron las leyes como nodos, mientras los enlaces representan el tipo de relación como modificación, implementación y sustitución legislativa. Acudiendo ya a las propiedades de la TdR aplicada para el análisis de redes legislativas, Boella et al. (2014) consideran que hay varias maneras en que esta contribuye:

a) Facilita la identificación de grupos de datos jurídicos: el trazado de los gráficos permite calcular el grado de distribución del número de vecinos de un nodo, lo que es denominado estructura comunitaria o aglomeraciones.

b) Posibilita el cálculo de la importancia de los nodos: este tiene en cuenta la dirección de la conexión entre los nodos que sirve para analizar la integridad del sistema. En el análisis realizado por Boella et al. (2014) se pone como ejemplo la modificación de una ley por otra cuya relación se da en un solo sentido, de esa manera el algoritmo utilizado por los sistemas de trazado de las redes permite calcular la importancia del nodo.

c) Permite realizar análisis de diámetro: en este caso, calcular el diámetro de una gráfica implica determinar el número de nodos que deben ser atravesados para viajar dentro de la red de un nodo a otro; en el caso de las redes legislativas, esta medida puede ser utilizada para estimar la complejidad de un sistema legal a partir de las sendas de citación y modificación.

Esta relación necesaria entre informática y derecho ha sido ya percibida por autores como Hamdaqa y Hamou-Lhadj (2011), quienes buscaron representar el sistema jurídico en forma de red de citación entre leyes, utilizando procesos de minería de datos para desarrollar una 
herramienta informática que sirva a las empresas para realizar consultas complejas respecto a la normatividad en distintos Estados y en relación con las normas internacionales en distintos sectores económicos. Un elemento crítico que aporta la perspectiva práctica de Hamdaqa y Hamou-Lhadj (2011) es la dificultad para interpretar grandes redes legales y donde se requiere continuar desarrollando herramientas para extraer información valiosa que los diseños informáticos actuales no permiten aún.

En un sentido similar, el texto de Koniaris et al. (2015) retoma las lecciones de los autores anteriores y dibuja una gran red legislativa de más de 60 años de antigüedad con un inmenso corpus legislativo de la Unión Europea en donde incluyeron tratados, leyes, regulación, decisiones de la Corte de Justicia; trazaron una red de 249690 nodos y 998902 arcos que los conectan. En esta red se puede ver la evolución de la legislación y simular el impacto de los cambios legislativos, para evaluar la resiliencia de las subredes legislativas.

De la misma forma que el análisis de redes permite visualizar sistemas jurídicos al interior de un Estado, también permite visibilizar las conexiones entre derechos nacionales y en relación con normativas supraestatales como las relaciones que se han formado en el caso de la Unión Europea. Para Losano (2005), en las sociedades postindustriales permanecen estructuras sociales heredadas de la sociedad industrial, por ello siguen siendo válidos los diseños legales y las explicaciones ius positivistas que fueron creadas en ese momento. No obstante, tiene problemas para explicar la coexistencia de los tres ordenamientos jurídicos existentes en la Unión Europea: los ordenamientos nacionales, los supranacionales y los internacionales, lo que ocasiona que la metáfora de la pirámide tenga aplicación en una parte del ordenamiento jurídico, pero para el resto es pertinente considerar el derecho como una red, retomando lo señalado por Ost y Kerchoven (2002).

En todos estos trabajos queda demostrada la pertinencia del análisis de redes en el estudio del sistema jurídico en su integridad, ya que permite la observación de grandes conjuntos de información en distintas ramas del derecho. Aun así, también muestra que todavía falta profundizar más en esta área, debido a que los estudios son pioneros en los distintos países y en la mayoría de los casos indican que 
las conclusiones y las utilidades de esta forma de observar el derecho hasta ahora están empezando a ser desarrolladas. Lo que sí se puede anticipar es que, como lo menciona Losano (2005), la representación piramidal del derecho va más allá de una estrategia de análisis o una descripción gráfica; por ejemplo, el modelo jerárquico en que se estructura el derecho tiene como consecuencia inmediata la existencia del Tribunal Constitucional, que puede eliminar cualquier ley que sea contraria a la Constitución y altere la coherencia del orden normativo, está por estudiarse a profundidad cuáles serían las consecuencias institucionales de considerar el derecho en red.

\section{TdR para analizar redes jurisprudenciales}

Al igual que se entrelazan cadenas de normas jurídicas, se entretejen las decisiones judiciales creando redes jurisprudenciales, campo en el cual varios investigadores han avanzado en estudios aplicados, debido a que la gran producción de decisiones y la citación entre sentencias construyen verdaderas redes de conocimiento, útiles para la investigación jurídica. Entre los estudios que aplican esta forma de analizar la jurisprudencia se encuentran Fowler et al. (2007), Winkels et al. (2011), Agnoloni \& Pagallo (2014), Whalen (2014a; 2014b), Kuppevelt y Van Dijck (2017) y Panagis y Tarissan (2017), en ellos se observa la utilidad de la TdR para manipular grandes cantidades de información y la posibilidad de hacer análisis a partir de los indicadores que arrojan los software estadísticos.

El texto pionero de Fowler et al. (2007) realiza una aplicación de análisis de redes. Los autores construyeron una red de 26681 decisiones del Tribunal Supremo de EE. UU. emitidas en el periodo 1791-2005, en el texto describen brevemente el método de red de citas entre sentencias y la puntuación de los precedentes más relevantes, para ello utilizaron las medidas de centralidad que ofrece la TdR. De esta manera, consideraron que la TdR aplicada a la conexión de casos permite revisar el desarrollo legal de un área de estudio. La medida de centralidad es importante en este caso porque es una forma de medir dentro de la TdR qué tan importante es un caso determinado en la Corte, además, sugieren que se puede utilizar para medir otros conceptos legales. 
En ese texto se evidencia el camino seguido por los autores para trazar la red, que es básicamente la ruta que debe atravesar cualquier investigador que quiera aplicar la TdR en investigaciones jurídicas. En primer lugar, acopiaron todas las decisiones de la Corte Suprema publicadas entre 1791 y 2005, con lo que obtuvieron las 26681 decisiones. En segundo lugar, identificaron las referencias entre las diferentes decisiones, con lo que mostraron cómo la técnica de TdR puede ayudar a medir la centralidad de las decisiones de la Corte Suprema.

De esta forma, Winkels et al. (2011) también realizaron un análisis de redes de citación jurisprudencial, pero en el caso holandés, en donde la tradición jurídica es de derecho continental, con el fin de determinar cuáles de las decisiones judiciales fueron más relevantes. Luego de realizar los cálculos de redes, validaron sus resultados con expertos en asuntos jurídicos, encontrando que la TdR aplicada a jurisprudencia es una herramienta útil para le investigación jurídica, incluso en donde el precedente judicial no es la fuente principal del derecho.

Del mismo modo, Whalen (2014a; 2014b) también aplica la TdR a las decisiones de la Corte Suprema de EE. UU., debido a que utiliza la base de datos de Fowler et al. (2007), dándole otros alcances investigativos. En este caso, enfatiza las posibilidades que ofrece la medida de centralidad y demuestra que las decisiones judiciales en las que la Corte Suprema realiza cambios importantes a los precedentes judiciales -que no son muy comunes en la historia jurisprudencial norteamericana-, tienen mayor centralidad, debido a que tienden a tener mayor citación. Así mismo, enfatiza que la TdR ofrece múltiples utilidades para la investigación.

Al igual que los investigadores anteriores, Agnoloni y Pagallo (2014) hacen una aplicación de análisis de redes a la jurisprudencia de la Corte Constitucional Italiana, para construir la red de citación de las sentencias de la Corte; en este caso también utilizaron las sentencias como nodos y las citaciones entre ellas como las aristas o líneas conectoras. El estudio involucra una gran cantidad de información, ya que implica las decisiones judiciales proferidas por este tribunal entre 1956 y 2013. Al construir la red de citación, los autores encontraron 16320 nodos y 57584 citaciones. Luego de trazar la red, procedieron a calcular la centralidad para revisar cuáles de las decisiones judiciales 
son más trascendentes. Su análisis arrojó los momentos de cambio de paradigma al interior de la Corte Constitucional y cuáles son las decisiones destacadas, como las relacionadas con los pronunciamientos de declaratoria de inconstitucionalidad de apartados del Código de Procedimiento Penal italiano.

Panagis y Tarissan (2017) utilizaron redes de citación explícitas o implícitas, es decir, también incluyeron las relaciones que se generan con similitud de textos al interior de la legislación europea y las decisiones del Tribunal de Justicia de la Unión Europea. En este caso, las redes son unidades textuales (párrafos). Este estudio es un paso metodológico importante en la integración de la TdR en estudios legales, porque tiene un análisis hermenéutico de los contenidos de los documentos involucrados en la red. El análisis realizado por Kuppevelt \& Van Dijck (2017) considera que las redes jurisprudenciales se posibilitaron a partir de la gran cantidad de información digitalizada que se encuentra en la actualidad, por ello desarrollaron un software de código abierto para trazar redes de decisiones judiciales en Holanda, para ser utilizado en la investigación jurídica. Sin embargo, son conscientes de que la investigación en esta materia apenas está empezando en la academia jurídica.

Finalmente, es importante anotar que las redes jurisprudenciales cumplen una función similar a las redes que representan los ordenamientos normativos, muestran el comportamiento de gran cantidad de información jurídica, en este caso de las preferencias y tendencias en decisiones judiciales permitiendo encontrar los puntos nodales que marcan la historia jurisprudencial.

\section{TdR para estructura interna de las constituciones políticas y las leyes}

En la aplicación de la TdR a un solo texto normativo como una constitución política o ley en particular, cada uno de estos es tomado como un corpus entero, en cuyo caso los investigadores modelan como un grafo el documento, lo que les permite visualizarlo e interpretarlo de una forma distinta a la hermenéutica tradicional. Este ejercicio es similar al realizado por los investigadores jurídicos en redes cuando miran las relaciones entre distintos cuerpos normativos para observar 
la complejidad del orden jurídico; en este caso se realiza a una escala microlegal, concentrándose en una sola norma y siguiendo la citación interna del documento.

En este campo, se destaca el trabajo de Bench-Capon et al. (1997) que explica los parámetros bajo los que se debe graficar la interrelación entre componentes de los documentos legislativos como una red donde los nodos son unidades textuales: "Cuando un documento se representa como un gráfico, el texto se divide en unidades significativas, que se convierten en los nodos del grafo, y los bordes representan relación entre estas unidades de texto" (p. 104).

Dicho de otra manera, cualquier ley puede ser considerada como un corpus jurídico y puede ser la base para trazar varios tipos de redes de acuerdo con los objetos que se definan como nodos y las relaciones entre ellos, que generalmente son de citación. Como se ha mencionado en varias oportunidades, los estudios aplicados de redes son los que demuestran las posibilidades que ofrece esta perspectiva, por eso es fundamental estudiar los textos que han utilizado este punto de vista, en donde se destacan Boulet et al. (2010), Winkels y Boer (2014), Sweeney et al. (2014), Karstens et al. (2014), Fitzgerald (2014), Tamme et al. (2014) y Lettieri et al. (2014b).

Una de las primeras investigaciones que se ha realizado bajo este enfoque es respecto al Código Ambiental francés, que realizaron Boulet et al. (2010), en donde tomaron como nodos cada uno de los artículos del código y los objetos de jerarquía interna del texto, como los capítulos o secciones en los que está dividido. Como relación para el trazado de los bordes se tuvieron en cuenta dos tipos de enlace: bordes tipo influencia, en donde se tiene en cuenta la pertenencia del artículo al libro o sección, y bordes de selección, que se refiere a la citación directa y explícita entre los artículos. Luego de trazar la red bajo estas premisas, y con el fin de buscar la estructura y las propiedades ocultas de la legislación ambiental estudiada, encontraron un grafo con 980 vértices y 2186 bordes a partir de los cuales se puede deducir que tiene las propiedades de pequeño mundo y está dividido en varias comunidades de artículos que solo coinciden parcialmente con la organización dada por la tabla de contenido de la norma. 
Un ejercicio similar al de Boulet et al. (2010) fue realizado por Winkels y Boer (2014), en el derecho alemán, quienes desarrollaron un ejemplo de construcción de red al interior de una ley a partir de expresiones contenidas en los artículos, retomadas por artículos subsiguientes. De esta manera se entreteje una compleja red conceptual a partir de referencias internas y externas del texto legal trazado, aclaran que las referencias internas pueden referirse a artículos anteriores o posteriores (anáfora y catáfora, respectivamente) y decidieron otorgar un mayor valor a las anáforas. Así mismo, incluyeron mayor ponderación de los conceptos repetidos frecuentemente en el texto legal; es decir, el número total de expresiones que existen de un trabajo pueden ser tomadas como un indicador de su relevancia. Finalmente, luego de trazada la red, calcularon la medida de centralidad con el número de referencias entrantes a un determinado nodo como la forma de clasificar los nodos en orden de importancia (grado); es decir, entre más conexiones tenga un nodo este es más importante.

En la misma línea de los autores anteriores, aparece el interesante trabajo de Sweeney et al. (2014), quienes crean una metodología para aplicar la TdR a la exploración de la estructura de las leyes estatutarias de once Estados al interior de EE. UU. En particular, se concentran en la legislación que regula el sistema de salud público en cada uno de los Estados y de esta forma proceden a realizar un ejercicio de forma comparada.

Por su parte, Karstens et al. (2014) realizaron una aplicación de la TdR a todas las constituciones políticas del mundo con el fin de mostrar la estructura interna de referencia de los textos como una forma para aportar a la interpretabilidad de los textos constitucionales. El punto de partida de esta investigación fue que es inusual que las cartas políticas de los Estados se sometan a investigaciones sistemáticas. Para ello trazaron las estructuras de referencia para todas las constituciones analizadas y, además de aplicar análisis de redes, calcularon la correlación interna de los textos teniendo en cuenta otros factores como longitud del texto, número de tópicos cubiertos e historia de colonización.

Lo que buscaron los autores de este estudio de constitucionalismo comparado fue medir la complejidad de las constituciones nacionales por medio del análisis de la estructura de referencia entre artículos del 
mismo texto, considerando estructuras de referencia como redes. La hipótesis de los autores era que los textos más complejos desde el punto de vista lingüístico deberían ser más difíciles de interpretar; es decir, la estructura de referencia de un texto se considera como una medida importante de legibilidad; por ejemplo, un texto que remita a muchos otros documentos indica que existe dificultad en la interpretación, y ponen como ejemplo el artículo 181.1 de la Constitución de Kenia de 1963, que remite al lector a seis secciones diferentes para calificar los poderes de la Corte de Apelación (Karstens et al., 2014).

Los resultados encontrados por los investigadores muestran dos grupos. En primer lugar, aquellos que tienen una enorme cantidad de referencias internas, que corresponden a países de la tradición del derecho consuetudinario, y en segundo, aquellos donde se tienen pocas o ninguna referencia, los países con una tradición de derecho civil. Muchas preguntas quedan abiertas de este estudio, entre ellas si hay un umbral de número de referencias que haga más o menos inteligible una constitución política. Sin embargo, queda claro que el uso de la visualización con redes permite la realización de estudios comparativos como lo confirman Tamme et al. (2014), quienes proponen un método de comparación de textos legales estonios utilizando un software. ${ }^{9}$

Un estudio similar a los registrados con anterioridad en este apartado es el de Fitzgerald (2014), quien presenta la aplicación del análisis de redes como una ayuda para la interpretación legal. Este autor considera que puede ayudar a los abogados a comprender mejor el contexto de una disposición y la estructura del Reglamento de Practicantes de Salud de la Ley Nacional de 2009 en Australia y se enfoca en las definiciones realizadas de esta ley para establecer las redes. Para el trazado, el autor acude al uso del software Pajek y a partir de los resultados realiza un análisis a nivel micro, concentrándose en la estructura molecular de la red analizada de forma que inicia graficando estructuras simples para luego proceder a redes más complejas de definiciones al interior de la ley. Con ello determinó qué tan sensible es la ley a los cambios, 
encontrando que la medida de centralidad arroja un indicador del impacto que tiene una modificación legal en el resto de la ley.

En esta sección de redes para representar la estructura interna de la ley queda evidenciado que hay muchos investigadores en la academia jurídica mundial que consideran válido el trazado de la distribución interna de los conceptos al interior de una norma. Así mismo, esto sirve para muchas cosas, entre ellas observar los conceptos que fungen como piedra angular en una norma para revisar la legibilidad e interpretabilidad de una constitución, para comparar el diseño de distintas normas ${ }^{10} \mathrm{y}$ para evaluar los impactos de los cambios normativos parciales en uno o varios artículos, entre otras aplicaciones.

\section{Límites y potencialidades de la TdR en los estudios jurídicos}

Como cualquier teoría, la TdR tiene sus limitaciones, algunas de las cuales seguramente el lector ya ha advertido. Una de ellas es que la posibilidad de analizar una gran cantidad de información puede convertirse en superficialidad en el análisis. Este aspecto es importante advertirlo antes de involucrar la TdR en una investigación, ya que no se debe considerar como una teoría para explicarlo todo:

su visión holística, el descubrimiento inesperado de semejanzas entre sistemas totalmente diferentes y la actual fascinación cultural conllevan la tentación de pensar que el análisis de redes es una 'teoría del todo'. Sociólogos, ingenieros, biólogos y filósofos han advertido contra generalizaciones simplistas que se obtienen de la ciencia de las redes (Caldarelli \& Catanzaro, 2012, p. 161).

No obstante, esa limitación es la misma que se puede predicar de una foto aérea o de un mapa que muestran las condiciones generales de una colectividad, pero no tienen como objetivo mostrar el detalle

10 Al respecto ver Cairns, 2013; Dawson, 1982; Watson, 1983a, 1993, 2001b, 2001a, 2007, y Zweigert y Kötz, 1998. 
de los objetos que contiene el conjunto que es fotografiado o dibujado; es decir, más allá de una limitación es una característica de la TdR que debe tener en cuenta el investigador que decide utilizarla para acercarse al objeto de estudio. Por ello no debe subestimarse el poder del análisis de redes, especialmente, sin explorar a profundidad primero el marco de la investigación jurídica. Es decir, si el objetivo es mostrar un panorama de una temática la TdR es útil, pero si el interés es revisar en detalle cada elemento, no es la mejor opción para el investigador o debe ser complementada con otro tipo de análisis.

Más que una limitación, hay un punto a tener en cuenta en las investigaciones que involucran redes, que puede potenciar o limitar los resultados que se obtengan: la definición de las aristas y el grado de relacionamiento. Es necesario definir el límite en el que se consideran relacionados dos elementos:

hay que establecer un umbral por debajo del cual se considera que la relación es demasiado débil para registrarse. Ponderar o poner un umbral en los enlaces influye enormemente en la forma de la red resultante y debe hacerse por buenos motivos (caldarelli \& catanzaro, 2012, p. 164).

Es decir, la claridad y exactitud del relacionamiento entre los vértices va a alterar la forma y los resultados de la visualización en red.

Una última limitación es su dependencia de las bases de datos, generalmente inexistentes o incompletas en materias jurídicas, que preferiblemente estén discriminadas por especialidades, con décadas de información recopilada y accesible desde plataformas digitales, como puede pasar con otras áreas del conocimiento.

Se considera que el estudio de redes es útil en la investigación jurídica, ya que permite acercarse a nuevas metodologías que han adoptado otras ciencias sociales y puede constituir una semilla próspera para aportar a la ampliación de las fronteras teóricas y metodológicas en el conocimiento jurídico.

Por todos los argumentos expresados a lo largo de este artículo se considera que puede haber un gran número de preguntas de investigación de índole jurídico que pueden ser contestadas mediante la TdR 
como una forma para avanzar en la forma en la que se estudian, que puede arrojar otro tipo de respuestas superando o complementando el estudio de la ciencia del derecho que ha estado anclado al uso de la interpretación como método fundamental para exponer ideas.

\section{Referencias}

Agnoloni, T., \& Pagallo, U. (2014). The case law of the Italian Constitutional Court between network theory and philosophy of information. En R. Winkels \& N. Lettieri (Eds.), Second International Workshop on Network Analysis in Law (pp. 11-38). http://www.leibnizcenter.org/ winkels/ NAiL2014-pre-proceedings.pdf

Ajani, G., Boella, G., Lesmo, L., Mazzei, R., Radicioni, D. P., G Rossi, P. (2008). Legal taxonomy syllabus: Handling multilevel legal ontologies. Proceedings of LangTech 2008, 3-6. http://citeseerx.ist.psu.edu/viewdoc/summary?doi $=10.1 .1 .110 .6427$

Arjona Sánchez, M. J. (2010). El derecho en red: una aproximación desde el derecho europeo. Videtur Quod, Anuario del Pensamiento Crítico, (2), 1-183. http://www.liberlex.com/archivos/derechoenred.pdf

Ashley, K., Ferrell, E., Potter, M., Guclu, H., Savelka, J., \& Grabmai, M. (2014). Statutory network analysis plus information retrieval. En R. Winkels \& N. Lettieri (Eds.), Second International Workshop on Network Analysis in Law (pp. 1-7).

Augsberg, I. (2009). The relevance of network models within the juridic discourse empirical, sociological, and epistemological perspectives. German Law Journal, 10(4), 383-394. https://static1.squarespace. $\mathrm{com} / \mathrm{static} / 56330 \mathrm{ad} 3 \mathrm{e} 4 \mathrm{~b} 0733 \mathrm{dcc} 0 \mathrm{c} 8495 / \mathrm{t} / 56 \mathrm{~b} 82 \mathrm{cfe} 746 \mathrm{fb} 90712 \mathrm{de}$ ab40/1454910718465/GLJ_Vol_10_No_04_Augsberg.pdf

Bench-Capon, T. J. M., Dunne, P. E. S., E Staniford, G. (1997). Modelling legal documents as graphs. Information \& Communications Technology Law, 6(2), 103-120. https://doi.org/10.1525/sp.2007.54.1.23

Boella, G., di Caro, L., Violato, A., \& Robaldo, L. (2014). Exploiting networks in Law. International Conference on Language Resources and Evaluation (LREC), 1654-1658. http://www.lrec-conf.org/proceedings/lrec2014/pdf/95_Paper. pdf 
Bommarito, M., \& Katz, D. (2010). A mathematical approach to the study of the United States Code. Physica A, 389(19), 4195-4200. https://doi. org/10.1016/j.physa.2010.05.057

Boulet, R., Mazzega, P., G Bourcier, D. (2010). Network analysis of the French Enviromental Code. AI Approaches to the Complexity of Legal Systems. Complex Systems, the Semantic Web, Ontologies, Argumentation, and Dialogue. Lecture Notes in Computer Science, 6237, 39-53. https://doi.org/10.1007/978-3642-16524-5

Boulet, R., Mazzega, P., E Bourcier, D. (2011). A network approach to the French system of legal codes-part I: Analysis of a dense network. Artificial Intelligence and Law, 19(4), 333-355. https://doi.org/10.1007/s10506-011-9116-1

Bourcier, D., \& Mazzega, P. (2007). Codification, law article and graphs. Legal Knowledge and Information Systems, JURIX 2007: The Twentieth Annual Conference, 29-38. https://www.researchgate.net/publication/220809998 Cairns, J. W. (2013). Watson, Walton, and the history of legal transplants. Georgia Journal of International and Comparative Law, 41(1), 637-696.

Caldarelli, G., \& Catanzaro, M. (2012). Redes: Una breve introducción. Alianza.

Dawson, J. P. (1982). Review: The making of the civil law. Alan Watson. Harvard University Press, Cambridge, 1981. Chicago Law Review, 1(49), 595-604. https://doi.org/10.1525/sp.2007.54.1.23

Fitzgerald, J. (2014). Network analysis as an aid to legal interpretation can counting and drawing rules help lawyers understand the context of those rules? En R. Winkels \& N. Lettieri (Eds.), Second International Workshop on Network Analysis in Law (pp. 72-82). http://www.leibnizcenter. org/ winkels/NAiL2014-pre-proceedings.pdf

Fowler, J. H., Johnson, T. R., Spriggs, J. F., Jeon, S., \& Wahlbeck, P. J. (2007). Network analysis and the law: Measuring the legal importance of precedents at the U.S. Supreme Court. Political Analysis, 15(3), 324-346. https:// doi.org/10.1093/pan/mpm011

Garbarino, C. (2014). A model of legal systems as evolutionary networks: Normative complexity and self-organization of clusters of rule. En R. Winkels \& N. Lettieri (Eds.), Second International Workshop on Network Analysis in Law (pp. 138-159).

Gultemen, D., \& van Engers, T. (2014). Graph-based linking and visualization for legislation documents (GLVD). En R. Winkels, N. Lettieri, \& S. Faro (Eds.), Network analysis in law (pp. 137-156). Edizione Scientifiche Italiane. Hamdaqa, M., \& Hamou-Lhadj, A. (2011). An approach based on citation analysis to support effective handling of regulatory compliance. Future 
Generation Computer Systems, 27(4), 395-410. https://doi.org/10.1016/j. future.2010.09.007

Hoekstra, R. (2014). A network analysis of Dutch regulations using the metalex document server. En R. Winkels, N. Lettieri, \& S. Faro (Eds.), Network analysis in law (pp. 95-112). Edizione Scientifiche Italiane.

Karstens, B., Koolen, M., Dari-Mattiacci, G., Bod, R., \& Ginsburg, T. (2014). Reference structures of national constitutions. En R. Winkels $\&$ N. Lettieri (Eds.), Second International Workshop on Network Analysis in Law (pp. 50-60).

Kelsen, H. (1982). Teoría pura del derecho. Universidad Nacional Autónoma de México.

Koniaris, M., Anagnostopoulos, I., \& Vassiliou, Y. (2015). Network analysis in the legal domain: A complex model for European Union legal sources. Journal of Complex Networks. https://doi.org/10.1093/COMNET/CNX029

Kuppevelt, D., \& van Dijck, G. (2017). Answering legal research questions about Dutch case law with network analysis and visualization. En A. Wyner \& G. Casini (Eds.), Legal knowledge and information systems (pp. 95-100). IOS Press.

Lettieri, N., Faro, S., Vicidomini, L., \& Altamura, A. (2014a). Nets of legal information connecting and displaying heterogeneous legal source. En R. Winkels \& N. Lettieri (Eds.), Second International Workshop on Network Analysis in Law.

Lettieri, N., Malandrino, D., Spinelli, R., \& Rinaldi, C. (2014b). From structure to function. Exploring the use of text and social network analysis in criminal investigations. En R. Winkels, N. Lettieri, \& S. Faro (Eds.), Network analysis in law (pp. 79-94). Edizione Scientifiche Italiane.

Lettieri, N., Malandrino, D., \& Vicidomini, L. (2014c). Document-enhacement and network analisys for criminal investigation: An holistic approach. En R. Winkels \& N. Lettieri (Eds.), Second International Workshop on Network Analysis in Law (pp. 16-38).

Losano, M. G. (2005). Derecho turbulento: En busca de nuevos paradigmas en las relaciones entre derechos nacionales y normativas supraestatales. DOXA. Cuadernos de Filosofía del Derecho, 28, 159-182.

Martin Katz, D., E Bommarito, M. (2011). Network analysis and law tutorial. http://computationallegalstudies.com/network-analysis-and-law-tutorial/ Mazzega, P., Bourcier, D., \& Boulet, R. (2009). The network of French legal codes. Proceedings of the 12th International Conference on Artificial Intelligence and Law (ICAIL '09), 236-237. https://doi.org/10.1145/1568234.1568271 
Mimouni, N., Nazarenko, A., E Salotti, S. (2014). An approach for searching and browsing a network of legal documents. En R. Winkels, N. Lettieri, $\mathcal{E}$ S. Faro (Eds.), Network analysis in law (pp. 183-208). Edizione Scientifiche Italiane.

Newman, M. E. J. (2010). Networks an introduction. Oxford University Press.

Ost, F., E van de Kerchoven, M. (2002). De la pyramide au réseau? Pour une théorie dialectique du droit. Publications des Facultés universitaires Saint-Louis.

Panagis, Y., E Tarissan, F. (2017). Giving every case its (legal) due. The contribution of citation networks and text similarity techniques to legal studies of European Union law. En A. Wyner \& G. Casini (Eds.), Legal knowledge and information systems (pp. 59-68). IOS Press.

Smith, T. A. C. (2005). The web of law. San Diego Legal Studies Research Paper, 6(11), 1-39. https://doi.org/10.2139/ssrn.642863

Sweeney, P., Bjerke, E., Potter, M., Guclu, H., Keane, C., Ashley, K., E Grabmair, Rebecca, H. (2014). Network analysis of manually-encoded state laws and prospects for automation. En R. Winkels, N. Lettieri, \& S. Faro (Eds.), Network analysis in law (pp. 53-78). Edizione Scientifiche Italiane.

Szöke, Á., Mácsár, K., E Strausz, G. (2014). Knowledge extraction framework for analyzing legal documents. En R. Winkels, N. Lettieri, E S. Faro (Eds.), Network Analysis in Law (pp. 113-136). Edizione Scientifiche Italiane.

Tamme, T., Vohandu, L., E Task, E. (2014). A method to compare the complexity of legal acts. En R. Winkels \& N. Lettieri (Eds.), Second International Workshop on Network Analysis in Law (pp. 105-114).

Täsk, E., Vohandu, L., Lohk, A., E Liiv, I. (2011). An experiment to find the deep structure of Estonian legislation. En K. Atkinson (Ed.), Legal knowledge and information systems (pp. 93-102). IOS Press.

Watson, A. (1983a). Legal change : Sources of law and legal culture. University of Pennsylvania Law Review, 131(5), 1121-1157.

Watson, A. (1993). Legal transplant: An approach to comparative law. University of Georgia Press.

Watson, A. (2001a). Society and legal change. Temple University Press.

Watson, A. (2001b). The evolution of western private law. The Jonhns Hopkins University Press.

Watson, A. (2007). Comparative law: Law, reality and society. Vandeplas $\mathrm{Pu}-$ blishing.

Watts, D. J., \& Strogatz, S. (1998). Collective dynamics of "small-world" networks. Nature, 393, 440-442. https://doi.org/10.1038/30918 
Whalen, R. (2014a). Bad law before it goes bad: Citation networks and the life cycle of overruled supreme court precedent. En R. Winkels, N. Lettieri, \& S. Faro (Eds.), Network analysis in law (pp. 1-22). Edizione Scientifiche Italiane. Whalen, R. (2014b). Citation networks and the life cycle of overruled Supreme Court precedent. En Radbound Winkels, L. Nicola, \& S. Faro (Eds.), Network analysis in law (pp. 1-22). Edizioni Scientifiche Italiane.

Winkels, R., E Boer, A. (2014). Finding and visualizing Dutch legislative context networks. En R. Winkels, N. Lettieri, \& S. Faro (Eds.), Network analysis in law (pp. 157-182). Edizione Scientifiche Italiane.

Winkels, R., de Ruyter, J., \& Kroese, H. (2011). Determining authority of Dutch case law. En K. Atkinson (Ed.), Legal knowledge and information systems (pp. 103-112). IOS Press.

Zhang, P., Silver, H., Wasson, M., Steiner, D., \& Saharma, S. (2014). Knowledge network based on legal issues. En Radboud Winkels, N. Lettieri, \& S. Faro (Eds.), Network analysis in law (pp. 23-52). Edizione Scientifiche Italiane.

Zweigert, K., \& Kötz, H. (1998). An introduction to comparative law. Oxford University Press. 